\title{
Some astrophysical implication of gas profiles in a new galaxy clusters model
}

\author{
Ester Piedipalumbo* \\ Dipartimento di Scienze Fisiche, Università Federico II di Napoli, Complesso Universitario di \\ MSA, Via Cinthia, 80126 Napoli, Italy \\ E-mail: esterena.infn.it
}

Cardone V.F.

Dipartimento di Fisica “E.R. Caianiello”, Università di Salerno, Via S. Allende, 84081 -

Baronissi (Salerno), Italy

Tortora C.

Dipartimento di Scienze Fisiche, Università Federico II di Napoli, Complesso Universitario di MSA, Via Cinthia, 80126 Napoli, Italy

\begin{abstract}
The structure of the dark matter and the thermodynamical status of the hot gas in galaxy clusters is an interesting and widely discussed topic in modern astrophysics. Recently, Rasia et al. (2004) have proposed a new dynamical model of clusters of galaxies, which describes both the dark matter halo and the hot intracluster gas. We analyze the influence of the gas density and temperature profiles on the properties of the X-emission and the comptonization parameter that determines the CMBR temperature decrement due to the Sunyaev - Zel'dovich effect!
\end{abstract}

Baryons in Dark Matter Halos

5-9 October 2004

Novigrad, Croatia

* Speaker.

${ }^{\dagger}$ A complete traetment of this topic is found in Cardone V.F., Piedipalumbo E., Tortora C., 2005, A\&A, 429, 49 


\section{The thermal Sunyaev - Zel'dovich effect}

At the beginning of 1970s, Sunayev and Zel'dovich ([6]) suggested that the cosmic microwave background radiation (CMBR) can be scattered by the trapped hot intracluster electrons giving rise to a measurable distortion of its spectrum. This (inverse) Compton scattering (now referred to as the Sunyaev-Zel'dovich effect, hereafter SZE) has been recognized in the last two decades as an important tool for cosmological and astrophysical studies ([1]). Because of this inverse Thomson scattering, an overall change in brightness of the CMBR is observed. In the non relativistic limit, the scattering process can be described by the Kompaneets equation

$$
\frac{\partial n}{\partial y}=\frac{1}{p_{e}^{2}} \frac{\partial}{\partial p_{e}}\left[p_{e}^{4}\left(\frac{\partial n}{\partial p_{e}}+n+n^{2}\right)\right]
$$

which describes the change in the occupation number of photons $n(v)$. Here $p_{e}=h v_{e} / k_{B} T_{e}$, while $y$ is the so called Comptonization parameter defined as :

$$
y \equiv \int \frac{k_{B} T_{e}}{m_{e} c^{2}} n_{e} \sigma_{T} d l
$$

where $\sigma_{T}$ is the Thompson scattering cross section and $n_{e}$ the electrons number density and the integral is performed along the line of sight. Assuming that the photons distribution after the scattering is close to the equilibrium one, and in the limit of low frequencies, we get the useful approximated expression :

$$
\frac{\Delta T}{T}=-2 y \text {. }
$$

Eq.(1.3) allows one to evaluate the shift in temperature due to the SZE provided that the gas number density $n_{e}(r)$ and the temperature profile $T_{e}(r)$ are given. For the RTM model, it is ([5]):

$$
n_{e}(s)=\frac{\rho_{g, 0} \rho_{b}}{\mu m_{p}}\left(s+x_{p}\right)^{-2.5}, T_{e}(s)=\frac{T_{0} T_{v} s^{0.016}}{\left(s^{4}+x_{p}^{4}\right)^{0.13}}
$$

where $s=r / R_{v}, \mu$ is the mean molecular weight, $m_{p}$ the proton mass, $T_{v}$ the virial temperature and $\rho_{g, 0}$ a normalization density given in Rasia et al. (2004).

Even if all of the physics of the effect is coded in the Compton $y$ parameter, it is the total flux density from the cluster that is used from the observational point of view. It is found by integrating the comptonization parameter over the cluster face obtaining $Y=\int y(\vec{\theta}) d^{2} \vec{\theta}$ where $\vec{\theta}$ is the angular position on the sky. If the gas temperature profile is isothermal, the integrated SZE flux may be simply related to the cluster temperature weighted by mass divided by $D_{A}^{2}$, where $D_{A}$ is the angular diameter distance. Actually, $d \Omega=d A / D_{A}^{2}$, and $Y \propto \frac{N_{e}\left\langle T_{e}\right\rangle}{D_{A}^{2}} \propto \frac{M\left\langle T_{e}\right\rangle}{D_{A}^{2}}$ where $N_{e}$ is the total number of electrons in the cluster, $\left\langle T_{e}\right\rangle$ the mean electron temperature, and $M$ the total mass of the cluster (or the mass of gas $\left.M_{g}=f_{g} M\right)$. However, for the RTM model, the temperature profile is approximately isothermal only in the inner region $\left(r \leq 0.2 R_{v}\right)$ so that the question of the detectability has to be significantly revised. Actually it turns out that the relative variation $\varepsilon_{Y}=1-Y_{R T M}^{i s o} / Y_{R T M}$ increases with the distance from the cluster centre being of order $25 \%$ at the virial radius. As a result, for a RTM cluster we have that $Y \propto \frac{M_{e f f}\left\langle T_{e}\right\rangle}{D_{A}^{2}}=\frac{v M\left\langle T_{e}\right\rangle}{D_{A}^{2}}$, where $v=Y_{R T M} / Y_{R T M}^{i s o}$. Because $v>1$, the SZE detectability of a RTM cluster is naturally improved: for a fixed threshold value $Y_{\text {lim }}$, it is possible to detect less massive clusters than in the isothermal case. 


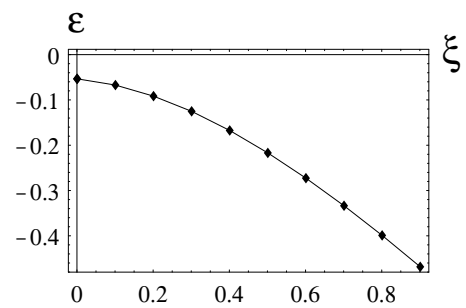

Figure 1: The relative error $\varepsilon$ on the structure integral $\eta$ vs $\xi$ due to the finite extension of the cluster. It is : $\varepsilon=[\eta(l \rightarrow \infty)-\eta(l=1)] / \eta(l=1)$.

\subsection{The gas profile and the structure integral}

The radial dependence of cluster profiles is becoming a testing ground for models of structure formation and for our understanding of gas dynamics in galaxy clusters. Actually, the formation of structures is believed to be driven by some hierarchical development, which leads to the prediction of self similar scalings between systems of different masses and at different epochs. From the observational point of view, however, the situation is rather controversial and yet undetermined : $\mathrm{X}$ rays observations of poor clusters fall below the self similar expectations, and even if the isothermal distribution is often a reasonable approximation of the actual observed clusters, some clusters show non isothermal distribution. It turns out that the emerging temperature profile is one where the temperature increases from the center to some characteristic radius, and then decreases again. On the other hand, the temperature profile described by Eq.(1.4) reproduces quite well some of these observational features : it shows an isothermal core up to $0.2 R_{v}$, followed by a steep decrease that reaches a factor of two lower around the virial radius; the density profiles are roughly self - similar for $s>0.06$, while the gas becomes flatter in the inner region. The observations of the SZE, which are becoming increasingly accurate, can be used to probe these properties. The temperature shift may be evaluated by inserting Eqs.(1.4) into Eqs.(1.3), thus obtaining :

$$
\frac{\Delta T}{T_{0}}=-\frac{2 k_{B} \sigma_{T} T_{e_{0}} n_{e_{0}}}{m_{e} c^{2}} \times \eta
$$

with $\eta$ the so called structure integral defined as $\eta=2 \int_{0}^{l} \frac{n_{e}(s)}{n_{e_{0}}} \frac{T_{e}(s)}{T_{e_{0}}} d l$ which depends only on the geometry and the extension of the cluster along the line of sight. The temperature profile of the RTM model is approximately isothermal up to $\sim 0.2 R_{v}$. Fig. 2 shows the structure integral $\eta$ vs $\xi$ for the RTM model using the correct temperature profile (upper curve) or the isothermality hypothesis. In particular, it turns out that in the inner region of the cluster, where the SZE effect is measured, $\eta_{\text {iso }}$ is more than $30 \%$ lower than the true $\eta$ thus leading to a similar error on the temperature shift. Finally, we analyzed also some possible geometrical effects on the structure intergral for the RTM model, as pointed out in ([4]): it turns out that neglecting the finite extension of the cluster systematically underestimates $\Delta T / T$ by an amount that is less than $10 \%$ in the inner regions of the cluster, where the SZE signal is usually detected (as shown in Fig 1). So that it may be neglected in a first order analysis.

\subsection{Comparison with standard gas profile}

In the present section we describe how the SZ signal from an RTM cluster compares to the SZ 


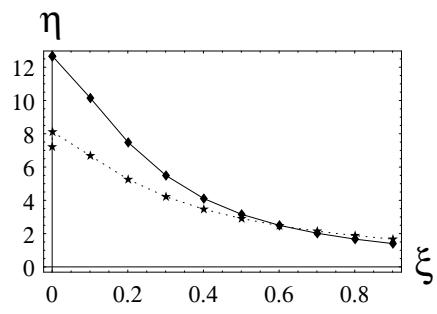

Figure 2: The structure integral $\eta$ vs $\xi$ for the RTM model using the correct temperature profile (upper curve) or the isothermality hypothesis (lower curve).

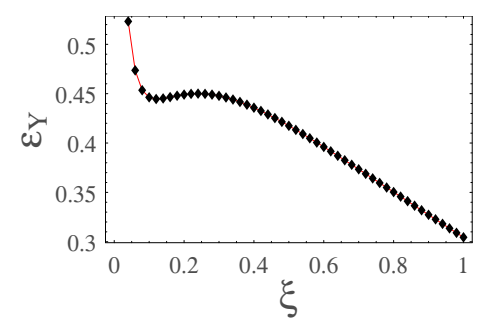

Figure 3: Relative deviation between the integrated SZE flux for a RTM an a $\beta$ model as function of the distance from the cluster center (in unit of $R_{V}$ )

signal from models with more standard temperature and density profiles: namely the $\beta$ model, and the NFW model.

\subsubsection{Comparison with a $\beta$ model}

The so called $\beta$ - model ([2]) is widely used in X-ray astronomy to parametrize the gas density profile in clusters of galaxies by fitting their surface profile: $\frac{\rho}{\rho_{b}}=\frac{\rho_{0 \beta}}{\left(x_{c}^{2}+x^{2}\right)^{\frac{3}{2 \beta}}}=\frac{\rho_{0 \beta} x_{c}^{-3 \beta}}{\left(1+\frac{x^{2}}{x_{c}^{2}}\right)^{\frac{3}{2 \beta}}}$, where $x_{c}$ is the core radius (measured in units of virial radius $\left(x_{c}=\frac{r_{c}}{R_{\nu}}\right), \rho_{0 \beta}$ is connected to the electron number density, $n_{e 0}$, at the cluster center $\left(n_{e 0}=\frac{\rho_{b} \rho_{0 \beta}}{\mu m_{p}}\right)$, and $\beta$ is a fitting parameter, which lies in the range $\frac{1}{2} \leq \beta \leq 1$. It turned out that, even if the density profiles of the models fit each other with good accuracy, the SZE signal is quite different, due to the different temperature profiles of the gas predicted for the two models. Actually the discrepancy is rather large also within the region $\xi \leq 0.8$, where the density profiles agree quite accurately. It implies that a SZE signal from a RTM cluster is larger than a signal from a $\beta$ cluster, still at large radius, and only in the extreme regions of the cluster it decreases $(\xi \geq 0.8)$. Moreover, in Fig. 3, we compare the relative deviation of the integrated SZE flux for the best fited $\beta$ model and a RTM model. It turns out that the integrated SZE flux is higher for a RTM cluster.

\subsection{The NFW model}

Even if it is mostly used to describe the dark matter rather than the gas distribution in galaxy clusters, it is nonetheless interesting to compare the SZE predictions for the RTM model with the same quantities evaluated for the NFW model.

As a first step, we fit the NFW model to the RTM one obtaining $c_{N F W}=5.99$ as best fit value for the concentration parameter. In agreement with Rasia et al. (2004), we find that the NFW model slightly overestimates the gas density in the range $0.04 \leq x \leq 0.4$, while it works sufficiently well in the outer regions. As shown in fig.4, we compare the SZE signal predicted from both models, plotting the relative discrepancy $\varepsilon_{y}=1-y_{N F W} / y_{R T M}$ between the comptonization parameters. We see that the SZE signal from a RTM cluster exceeds that from a NFW model as yet observed when comparing to the $\beta$-model. The RTM model thus emerges as the most effectively detectable also 


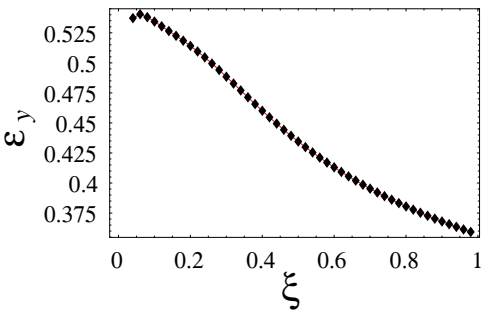

Figure 4: Relative discrepancy between the Componization parameter for a RTM model and its best fitting NFW model.

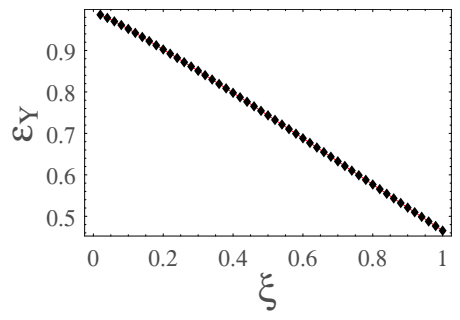

Figure 5: Relative discrepancy between the integrated SZE fluxes for a RTM model and its best fitting NFW model.

at larger distances from the cluster centre. This is yet more clear from Fig. 5 where we compare the integrated SZE flux for the RTM and NFW models.

\section{A short comment on possible systematic errors}

There is a potential caveat about the RTM model that could affect the main results we have discussed insofar. The simulations that have been considered by Rasia et al. (2004) to develop the model are based non radiative hydrodynamics so that cooling flows and cold blobs that may eventually be along the line of sight are not reproduced. Taking into account these effects is a difficult task, but we expect, qualitatively, that cooling flows and conduction should lower the central temperature and thus increase the SZE signal at the centre. However, we stress that detailed simulations also taking into account star formation and feedback processes are needed to investigate how the gas temperature profile (and thus the SZE signal) are affected. Some preliminary results have been presented ([5]), but the effect on the SZE flux has still to be investigated.

As a final remark, it is worth noting that merging of clusters has not been considered, but it is likely that this does not affect the main results. Actually, the sample of simulated clusters analyzed by Rasia et al. (2004) comprises both relaxed, unrelaxed and post-merging systems and the RTM model turns out to be a good fit to the full sample which is an evidence strongly suggesting that merging effects do not alter significantly the cluster structure. As a result, the clusters properties connected to the dark matter component, as for example the lensing properties, of the model are likely to be affected only when the merging is in progress in which case an external shear could mimic to first order the deviations from spherical symmetry of the outer regions of the dark matter halo. A stronger effect is expected for the impact of merging on the SZE signal and the $\mathrm{X}$-ray emission, since they depend on $n_{e_{0}}$ and $n_{e_{0}}^{2}$ respectively. Although further detailed simulations are needed to quantitatively address this question, some partial analytical results have been already obtained for some special mergers regimes, when the presence of cold fronts marks the late merging stages: namely the transonic and the subsonic mergers. It turns out that in the transonic regime the frequency spectrum of the SZE signal $g(p)$ changes, due to a shock particle re acceleration mechanism, depending on the concentration, which induces a new electron population. As net effect, the crossover changes still up to $\sim 10 \%$. In the subsonic case, instead, $g(p)$ remains unchanged, but the amplitude of the SZE signal is enhanced in a not negligible way, mainly in the interior regions of the cluster where it reaches also $\sim 30-40 \%$ ([4]). Moreover, it is well known 
that the $\mathrm{X}$ - ray luminosity overall increases during merging so that it is likely that, as net effect, the SZE signal is enhanced during cluster merging events.

\section{Conclusions}

Being detectable at high redshift, galaxy clusters are promising tools for determining cosmological parameters and testing theories of structures formation. Hydrodynamical simulations are able to predict not only the dark matter mass distribution, but also the density law and the temperature profile of the gas component thus allowing a study of the Sunyaev - Zel'dovich effect due to the cluster. One of the most interesting feature of the RTM model is that it allows to describe not only the dark matter, but also the hot intracluster gas component, providing analytical forms for its density, velocity and temperature profiles. We have investigated the implications of the RTM model for the intracluster gas on the SZE evaluating the structure integral that determines the temperature decrement. We have thus taken into account both the finite extension of the model and its peculiar temperature profile estimating the errors induced by the usually adopted simplifications of infinite extension and isothermal temperature. The main results are as follows.

1. Neglecting the finite extension of the cluster systematically underestimates $\Delta T / T$ by an amount that is less than $10 \%$ in the inner regions of the cluster so that it may be neglected in a first order analysis.

2. Using $T=T_{e, 0}$ as temperature profile instead of that found by RTM leads to underestimate $\Delta T / T$ up to $30 \%$ (in absolute value) in the centre.

3. The comptonization parameter y for the RTM model is higher than that of both the $\beta$ and the NFW models even if the parameters are chosen in such a way that the gas density is well fitted by the three models. Using the best fitting $\beta$ (NFW) model instead of the correct RTM one underestimates y up to $\sim 40 \%$ ( $\sim 2 \%$, respectively) in the inner cluster regions.

4. The non isothermal temperature profile leads to an integrated SZ flux which is higher for the RTM model than for both the $\beta$ and NFW models by an amount that depends on the distance from the cluster centre, but can be as high as $\sim 45 \%$ and $\sim 90 \%$ for the $\beta$ and NFW models respectively. As a result, less massive clusters should be detected in SZE survey if the RTM model is indeed the correct one.

\section{References}

[1] Birkinshaw, M. 1999, Phys. Rept., 310, 97

[2] Cavaliere, A., Fusco Femiano, R., 1976, A\&A, 49, 137

[3] Navarro, J.F., Frenk, C.S., White, S.D.M. 1997, ApJ, 490, 493

[4] Piffaretti, R., Jetzer, Ph., Schindler, S. 2003, A\&A, 398, 41; Koch, P., Jetzer, Ph. 2004, astro - ph/0406461

[5] Rasia, E., Tormen, G., Moscardini, L. 2004, MNRAS, 531, 237; Dolag, K., Jubelgas, M., Springel, V., Borgani, S, Rasia, E. 2004, ApJL, 606, 97

[6] Sunyaev, R., Zel'dovich, Ya., B. 1970, Comm. Ast. Sp. Ph., 2, 66; Sunyaev, R., Zel'dovich, Ya., B. 1972, MNRAS, 292, 159 